\title{
Realismus statt Worst Case
}

\section{Selbstverständlich schafft Windenergie Arbeitsplätze. Betrachtet man die Stu- die des bremer energie institutes näher, so zeigen sich einige methodische Schwächen und wenig plausible Annahmen. Von einer "nüchternen Analyse” kann deswegen nicht gesprochen werden.}

D re per Saldo zu Beschäftigungsverlusten und den Angaben von Verbandsvertretern und Bundesumweltministerium zu Arbeitsplätzen im Bereich erneuerbarer Energien sei nicht zu trauen - Dies sind die Hauptbotschaften einer Studie des bremer energie instituts zu den Beschäftigungswirkungen im Bereich erneuerbarer Energien. Die tiefere Betrachtung dieser Studie verdeutlicht, dass diese Aussagen in starkem Maße dadurch bestimmt werden, dass

- eine Methodik gewählt wird, die wesentliche dynamische Effekte ausklammert,

- unplausible Annahmen für die Modellrechnungen gesetzt werden, die von den Autoren zum Teil selbst als Worst Case bezeichnet werden und

- die ermittelten Ergebnisse einseitig interpretiert werden.

Die Zuspitzung dieser Ergebnisse ist umso erstaunlicher, als dass die insgesamt vermutlich eher deutlich positiven Gesamtbeschäftigungseffekte des Umbaus des deutschen Energiesystems in Richtung Nachhaltigkeit, Klimaschutz und Atomausstieg auch vom bremer energie institut bisher nicht bestritten werden.

\section{- Problematisches Vorgehen}

Das vom bremer energie institut verwendete InputOutput-Modell vernachlässigt die folgenden dynamischen Effekte:

- Einkommensmultiplikator-Effekte der Investitionen in erneuerbare Energieanlagen und ihres Betriebs, die sich positiv auf den Gesamtbeschäftigungseffekt auswirken würden;

- von verschiedensten Experten erwartete Preissteigerungen bei den fossilen Energieträgern bei zugleich sinkenden Preisen für erneuerbare Energien in Folge deren zunehmender Marktdurchdringung in den nächsten Jahren. Diese Entwicklungen würden zu einem Schrumpfen des so genannten Budgeteffekts, der Differenz aus Vergütung und Marktwert der Energie, führen, der ausschlaggebend für den vom bremer energie institut ermittelten negativen Gesamtbeschäftigungseffekt ist;

- mögliche Anpassungsreaktionen auf Energiepreisänderungen wie zum Beispiel verstärkte Energieeinspar-Aktivitäten industrieller Abnehmer;

- Steigerungen der Exportmöglichkeiten durch Entwicklung innovativer erneuerbarer Energietechnologien.

\section{Unplausible Annahmen}

Aus einer Bandbreite möglicher Annahmen für die vorgenommenen Modellrechnungen werden Extrema ausgewählt, die dazu führen, dass insgesamt wie von den Autoren bei einzelnen Annahmen betont - ein Worst Case-Szenario gebildet wird. Sensitivitätsanalysen werden kaum vorgenommen, obwohl bereits bei gering abweichenden, aus unserer Sicht realistischeren Annahmen positive Gesamtbeschäftigungseffekte abgeleitet werden können. Unwahrscheinlich beziehungsweise unplausibel sind insbesondere die folgenden Annahmen:

- Wind- und PV-Strom werden vermiedene Kosten in Höhe von $1 \mathrm{Cent} / \mathrm{kWh}$ zugerechnet, was noch unter den vermiedenen Brennstoffkosten konventioneller Kraftwerke liegt. Hier werden die Mehrkosten der Regelenergie überschätzt, der Beitrag der Windenergie zur Leistungseinsparung in einem intelligenten Strom-Mix unterschätzt und zu erwartende Energiepreissteigerungen vernachlässigt. Für den Fall, dass man dem Windstrom, wie von den Autoren selbst in einer Sensitivitätsrechnung vorgeschlagen, den plausibleren Wert von 3,0 Cent $/ \mathrm{kWh}$ (durchschnittlicher Börsenpreis) zurechnet, errechnen die Autoren per Saldo einen deutlich positiven Gesamtbeschäftigungseffekt.

- Bei nur um geringfiugig das heißt einige wenige Prozentpunkte niedrigeren Annahmen für die Vergütungssätze für den EEG-Strom kehren sich die Ergebnisse schon um, was die hohe Sensibilität von den Ausgangsparametern zeigt.

- Der angenommene Zertifikatspreis von fünf Euro pro Tonne Kohlendioxid liegt am unteren Ende gängiger Abschätzungen und deckt bei weitem nicht die der konventionellen Stromerzeugung eigentlich zuzurechnenden externen Kosten.

\section{Unzulässige Interpretationen}

Die Ergebnisse werden zum Teil in einer Art und Weise interpretiert, die den Eindruck erweckt, die Studie diene weniger dem Erkenntnisfortschritt als vielmehr als gezielt eingesetztes Instrument zur Legitimation bestimmter energiewirtschaftlicher Interessen und zur Abwehr einer weitergehenden Förderung erneuerbarer Energien. Hierzu zwei Beispiele: Die Studie konstruiert erstens einen generellen Widerspruch zwischen den Arbeitsplatzangaben des Bundesumweltministeriums (BMU) beziehungsweise den Angaben von Interessenverbänden einerseits und den erzielten telefonischen Umfrageergebnissen andererseits, um die BMU- und Verbandsangaben grundsätzlich in Frage zu stellen, obwohl

- eine signifikante Abweichung nur im Teilsegment Windenergie identifiziert wird,

- die Umfrage von den Autoren als ,weder vollständig noch repräsentativ" bezeichnet wird,

- bei den Verbandsangaben wie auch bei Zahlen, die das Bundesumweltministerium im Jahr 2002 publiziert hat, im Einzelnen unklar bleibt, inwieweit Arbeitsplatzeffekte der Vorleistungsketten in die Istwerte hineingerechnet wurden.

Die Darstellung der Beschättigungseffekte basiert zweitens auf der Annahme eines konstanten Ausbaus über 20 Jahre. Wird dieser Ausbau abrupt beendet, kommt es zwangsläufig dann nur noch zu negativen Budgeteffekten, während positive Investitionseffekte ausbleiben. Mit dem real zu erwartenden Verlauf hat eine derartige Annahme allein wegen der vermutlich erfolgenden Ersatzinvestitionen (Repowering) wenig zu tun.

Insgesamt wird die Studie damit ihrem selbst gesteckten Anspruch, zu ,einer nüchternen und sachgerechten Einschätzung der durch die Förderung der Nutzung Erneuerbarer Energien zu erzielenden Beschäftigungseffekte" zu kommen, nicht gerecht. Schade.

\section{Die Autoren}

Wolfgang Irrek ist wissenschaftlicher Mitarbeiter, Dr. Manfred Fischedick Forschungsgruppenleiter am Wuppertal Institut für Klima, Umwelt, Energie. Kontakt: Wuppertal Institut, Döppersberg 19, 42103 Wuppertal. E-Mail: wolfgang.irrek@wupperinst.org, manfred.fischedick@wupperinst.org 
(c) 20I0 Authors; licensee IÖW and oekom verlag. This is an article distributed under the terms of the Creative Commons Attribution Non-Commercial No Derivates License (http://creativecommons.org/licenses/by-nc-nd/3.o/), which permits unrestricted use, distribution, and reproduction in any medium, provided the original work is properly cited. 